\title{
Review
}

\section{Interleukin-21: a multifunctional regulator of immunity to infections}

\author{
John S. Yi, Maureen A. Cox, Allan J. Zajac* \\ Department of Microbiology, University of Alabama at Birmingham, 845 19th Street South, BBRB 446; Box 23, Birmingham, AL 35294-2170, United States
}

Received 10 August 2010; accepted 24 August 2010

Available online 9 September 2010

\begin{abstract}
Interleukin-21 (IL-21) is a cytokine that has broad effects on both innate and adaptive immune responses. The roles of IL-21 in determining immunity to infections are currently being defined, and notably, it has been shown that IL-21 is most critical for sustaining $\mathrm{T}$ cell responses during chronic viral infections. This article discusses our current understanding of the immunobiology of IL-21, as well as its known and potential roles in influencing immunity to infections.

(C) 2010 Institut Pasteur. Published by Elsevier Masson SAS. All rights reserved.
\end{abstract}

Keywords: Interleukin-21; Immunity; Infection

\section{Introduction}

Infections trigger multiple components of the host's immune response. The coordinated actions of these various cell types and soluble mediators are instrumental in determining the severity and outcome of the infection. As with all complex processes, regulatory mechanisms operate at every stage. This allows effective mobilization of the response, as well as typically ensures that homoeostasis is restored as the infection is either cleared or establishes persistence within its new host. Interleukin-21 (IL-21) is a member of the common$\gamma$ chain $(\gamma c)$ family of cytokines which also includes IL-2, IL4, IL-7, IL-9, and IL-15 (reviewed in Refs. [1,2]). As a consequence of its ability to act on multiple cells of the immune system, IL-21 has the potential to impact both innate and adaptive immune responses. Consistent with these broad actions, IL-21 has been shown to influence autoimmunity, tumor-specific responses, and also immunity to infections. In this review we summarize the biology of IL-21 and overview its impact on immune responses, highlighting the significance of IL-21 in protection against pathogens.

\footnotetext{
* Corresponding author. Tel.: +1 205975 5644; fax: +1 2059755482. E-mail address: azajac@uab.edu (A.J. Zajac).
}

\section{Interleukin-21 and its receptor}

The cloning of the interleukin-21 receptor (IL-21R), also originally termed novel interleukin receptor, was an essential first step that allowed the identification of its ligand, IL-21 [3,4]. The human IL-21R is encoded within an approximately $20 \mathrm{~kb}$ region on chromosome 16, adjacent to the IL-4R $\alpha$ gene. IL-21R is a 538 amino acid protein most closely related to IL-2R $\beta$ but also shares similarity with IL-4R $\alpha$, suggesting that it may have arisen by gene duplication. Comparison of human IL-21R with its murine ortholog, encoded on chromosome 7 , revealed that these gene products share $62 \%$ amino acid sequence similarity. The extracellular regions contain two pairs of conserved cysteine residues and a tryptophan-serine-X-tryptophan-serine (WSXWS) motif $[3,4]$. Potential signaling properties are also indicated by the primary sequence of the cytoplasmic domain which features partially conserved box 1 and box 2 domains, six tyrosine residues, and a conserved signal transducer and activator of transcription (STAT)-3 binding site [3,4]. The distinct IL-21R chain couples with the $\gamma c$ and together form the cytokine receptor complex [5]. Although IL-21 has been shown to bind to IL-21R in the absence of the $\gamma \mathrm{c}$, intracellular signaling only proceeds if the $\gamma c$ chain is present.

Analyses of the tissue distribution of the IL-21R by northern blot determined that it was predominately expressed 
in the spleen, thymus, peripheral blood, and lymph nodes $[3,4]$. Weaker hybridization was also detected in the lung and small intestine, which is likely due to the presence of lymphocytes at these sites. Further inspection of IL-21R expression patterns have shown that it is constitutively expressed by B cells and natural killer (NK) cells, but on T cells it is upregulated following activation. This suggests that any actions of IL-21 on T cells may be temporally regulated and dependent upon the activation status of the responding cell. IL-21R is also present on additional cell types including dendritic cells (DCs), macrophages, fibroblasts, and epithelial cells [6-9]. This expression pattern is consistent with the documented broad immunological actions of IL-21.

IL-21 is encoded on chromosomes 3 and 4 in mice and humans, respectively, and is closely genetically linked to IL-2. The mature human IL-21 polypeptide is 131 amino acids in length and shares 57\% amino acid identity with its 122 residue murine counterpart [4]. It is predicted to form a four- $\alpha$-helix bundle typical of type I cytokines and is most similar to IL-2, IL-4, and IL-15. IL-21 is not detected in normal tissues, but upon activation is most notably expressed by CD4 T cells [4]. IL-21 has also been shown to be produced by natural killer T (NKT) cells and there are indications that it can also be expressed by CD8 T cells under certain conditions (see Fig. 1) [10-12].

As with other $\gamma c$ cytokines, the engagement of IL-21 with its receptor complex activates Janus tyrosine kinases (JAKs), with the IL-21R chain inducing JAK1 phosphorylation and JAK3 interacting with the $\gamma_{\mathrm{c}}[3,5]$. Downstream of JAK recruitment, IL-21 primarily activates STAT3, although it can also trigger STAT1 and weakly activate STAT5 [5]. These signaling pathways ultimately modulate the transcription program of the activated cell, regulating its phenotype and fate. Transcription factors controlled by IL-21 include B-cell lymphoma 6 (Bcl-6), transcription factor 7 (Tcf7), and lymphoid enhancer binding factor 1 (Lef1), which are expressed in cells that are less terminally differentiated and maintain their proliferative capacity; as well as B-lymphocyte induced maturation protein 1 (Blimp-1) and c-Maf, which are associated with terminal differentiation and effector functions [13-16].

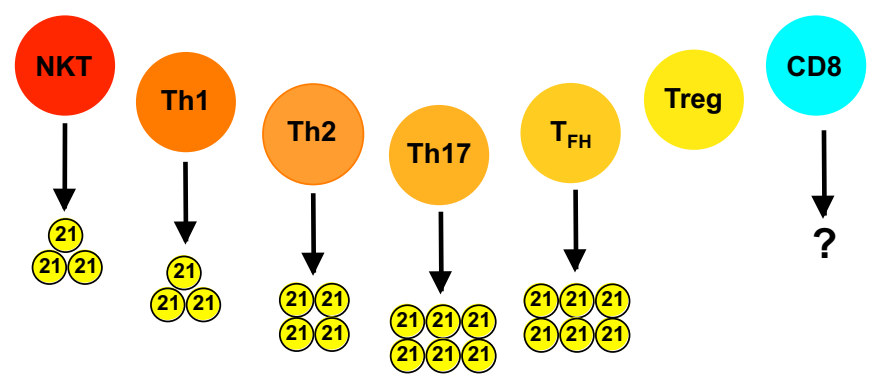

Fig. 1. The sources of IL-21. Both NKT cells and CD4 T cells have been shown to produce IL-21. Within the CD4 T cell population, Th17 and Tfh are the most prominent producers, whereas IL-21 is not secreted by CD4 Treg cells. CD8 $\mathrm{T}$ cell responses are not commonly associated with IL-21 production, although a limited number of studies indicate that this can occur.

\section{IL-21 and innate immunity}

As illustrated in Fig. 2, IL-21 regulates numerous aspects of innate immune responses. The effects of IL-21 on the individual cellular subsets which contribute to these responses are summarized below.

\subsection{Dendritic cells}

DCs play a pivotal role in triggering immune responses following infection. Exposure to $\gamma c$ cytokines such as IL-15, and also lipopolysaccharide promote the DC maturation, which is characterized by the upregulation of major histocompatibility complex (MHC) class II glycoproteins and costimulatory molecules. By contrast, IL-21 inhibits this differentiation process by reducing the expression of MHC class II, CD80, CD86, C-C chemokine receptor 7 (CCR7), and the production of cytokines including IL-6, IL-12, IL-1 $\beta$, and tumor necrosis factor- $\alpha$ (TNF- $\alpha$ ), while enhancing the uptake of antigen $[6,17]$. The reduction in cytokine production could be attributed to the expression of suppressor of cytokine signaling- 1 and -3 which are upregulated upon IL-21 stimulation [17].

\subsection{Natural killer cells}

NK cells provide an important first line of defense against many pathogens. IL-21 is not required for the development of murine NK cells; however, it does cause these cells to become more granular, upregulate perforin expression, and increase interferon- $\gamma($ IFN- $\gamma)$ production, thereby promoting effector activities [18,19]. In human NK cells, IL-21 can also enhance IFN $-\gamma$ production, proliferation, cytotoxicity, and accelerate maturation, as well as alter NK cell receptor expression [4,20-22].

\subsection{Natural killer T cells}

NKT cells are innate-like $\mathrm{T}$ lymphocytes that recognize glycolipid antigens in the context of the MHC class I-like molecule, CD1d. Although their antigen recognition is limited, NKT cells are important mediators in autoimmunity, infections, and cancer. Stimulation of NKT cells with IL-21 and $\alpha$-GalCer induces perforin production and enhances protection against tumors [23]. IL-21 also promotes the survival of NKT cells, although it is less effective than IL-2 and IL-15; however, IL-21 signaling in the presence of IL-2 or IL-15 causes NKT cells to become more granular and express higher levels of granzyme B [10]. Significantly, NKT cells have been shown to produce IL-21 in vitro following stimulation with anti-CD3 and -CD28 antibodies and also in vivo following $\alpha$ GalCer injection [10]. Thus, autocrine production of IL-21 may further activate the responding NKT cells. During infections it is plausible that NKT cells temporally complement CD4 $\mathrm{T}$ cells as a source of IL-21. 


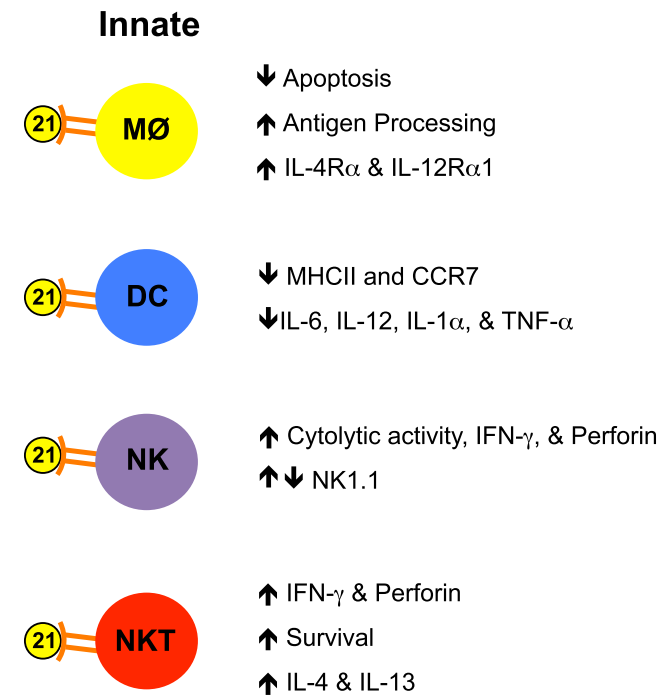

Adaptive

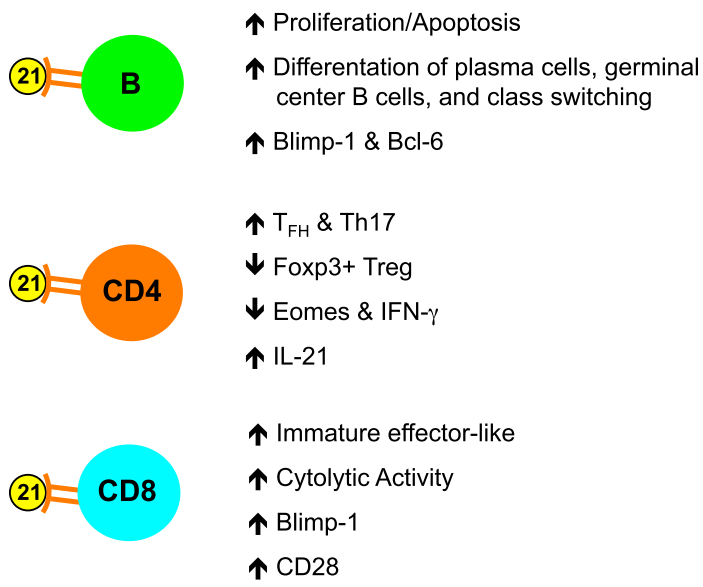

Fig. 2. The influence of IL-21 on the differentiation, function, and survival of cells of the immune system. IL-21 enhances the function of macrophages, NK, and NKT cells. It also supports the formation of germinal center B cells and the generation of plasma cells. IL-21 promotes the differentiation of Th17 cells and sustains Tfh responses but inhibits the development of Tregs. Additionally, IL-21 can restrict the maturation of DCs and may limit the differentiation of effector CD8 T cells.

\subsection{Other cell types}

Macrophages phagocytose extracellular pathogens and apoptotic cells, present antigens to $\mathrm{T}$ cells, and produce inflammatory mediators. IL-21 activates the phagocytic and proteolytic activities of macrophages. It also has a prosurvival role by preventing apoptosis through upregulation of the cyclin-dependent kinase inhibitors $\mathrm{p} 21^{\text {waf1 }}$ and $\mathrm{p} 21^{\mathrm{kip} 1}$ [9].

Matrix metalloproteinases (MMPs) are a group of neutral endopeptidases that are capable of degrading the extracellular matrix (ECM), and increased levels of MMPs have been observed following bacterial and viral infections, and also during autoimmune diseases. IL-21 can enhance the production of MMP-1, MMP-2, MMP-3, and MMP-9 by fibroblasts, and MMP-2 and MMP-9 by gastric epithelial cells [7,8]. Thus release of MMPs induced by IL-21 may contribute to extravascularization, inflammation, and remodeling of the ECM.

\section{IL-21 and adaptive immunity}

IL-21 not only influences innate immune responses but also affects the differentiation, acquisition of effector traits, and maintenance of both $\mathrm{B}$ and $\mathrm{T}$ cell responses (see Fig. 2).

\subsection{B cells}

B cells contribute to immunity to infections by serving as antigen-presenting cells and, most significantly, by giving rise to pathogen-specific antibodies. Although IL-21 is not required for B cell development, it does induce death, proliferation, and class switching in mature B cell populations, depending upon the antigenic stimuli and provision of accessory signals [24,25]. Without IL-21 the antibody isotype distribution is disrupted; reduced levels of $\operatorname{IgG} 1, \operatorname{IgG} 2 \mathrm{~b}, \operatorname{IgG} 3$, and increased levels of IgE are detected [26]. Germinal center (GC) development does not require IL-21; however, IL-21 maintains these reactions by regulating Bcl-6 expression [27-30]. IL-21 also controls several genes that dictate plasma cell formation and has been shown to upregulate Blimp-1, while decreasing paired box 5 (Pax 5) levels [31-33]. Plasma cell numbers are increased by the addition of IL-21 but are lower if IL-21 is absent [31,33]. Thus, IL-21 influences several aspects of $\mathrm{B}$ cell and antibody responses which have the potential to impact short- and long-term infection control.

\subsection{CD4 T cells}

CD4 $\mathrm{T}$ cells give rise to diverse and functionally distinct subsets which play key roles in autoimmunity, helping antibody and CD8 $\mathrm{T}$ cell responses, and promoting anti-pathogen activities. CD4 T cells are a principle source of IL-21, and this cytokine also influences the emergence of effector CD4 T cell subsets [4]. IL-21 has been reported to contribute to the development of $\mathrm{T}$ helper 17 (Th17) cells by upregulating expression of the IL-23R, which is critical for Th17 lineage commitment [34]. In the absence of IL-21, the generation of Th17 cells is impaired [35-37]. Th17 cells can also produce IL-21; this is greatly enhanced by IL-6, but is blocked in a dose dependent manner by TGF- $\beta$ [38]. The expression of IL-2 1 by Th17 cells also acts in an autocrine manner to amplify its own synthesis. In addition to driving Th17 development, IL-21 also inhibits the development of forkhead box $\mathrm{P} 3+$ (Foxp3) regulatory CD4 $\mathrm{T}$ cells (Treg), which can suppress both autoimmune responses as well as responses to pathogens $[35,36,39]$.

Although various lineages of CD4 T cells are capable of producing IL-21, a major source of this cytokine are $\mathrm{T}$ follicular helper cells (Tfh), which localize to B cell follicles [40]. Within the follicles they supply the responding B cells with IL-21. Initially, IL-21 was suggested to be required for the generation of Tfh cells; however, under certain conditions 
both Tfh cells and GC can form in the absence of IL-21 but are short-lived, suggesting a mutual dependence of Tfh and GC B cells $[29,30,41,42]$. High levels of inducible T-cell co-stimulator (ICOS) on Tfh cells allow interaction with ICOS ligand expressing B cells and costimulation of the Tfh cells. ICOS signaling has been shown to upregulate the transcription factor c-Maf, which in turn promotes IL-21 expression [15]. IL-21 can also induce c-Maf, providing a feedback mechanism to maintain IL-21 expression in Tfh cells. Tfh cells can coproduce IL-21 and IFN- $\gamma$, IL-4, or IL-17 depending upon whether there is polarization towards Th1, Th2, or Th17 response $[15,43,44]$.

\section{3. $C D 8 T$ cells}

CD8 $\mathrm{T}$ cells play a vital role in providing immunological protection against intracellular pathogens. The generation of effector and memory CD8 T cells is governed by the strength of TCR signaling and costimulation, as well as by the composition of the cyokine milieu. IL-21 has been shown to have both a direct and indirect influence on CD8 $\mathrm{T}$ cell responses, and has been proposed to act as a "third signal" to promote CD8 T cell activation [45]. The unique roles of IL-21 on CD8 T cell differentiation are highlighted by studies which show that CD8 T cells stimulated in the presence of IL-21 are phenotypically distinct from cells activated by IL-12, IFN- $\alpha$, IL-2 and IL-15 [14,45,46]. IL-2 and IL-15 promotes the generation of mature effector CD8 T cells by augmenting proliferation, killing ability, and the expression of CD44, CD25, IFN- $\gamma$, granzyme B, and eomesodermin (Eomes). In contrast, antigen-activated CD8 T cells stimulated with IL-21 express lower levels of CD44, CD25, Eomes, granzyme B, IFN- $\gamma$, and show decreased killing ability but express higher levels of Tcf7, Lef1, and CD62L [14]. Separate studies also show that IL-21 can positively impact the production of IL-2 by the responding CD8 $\mathrm{T}$ cells $[47,48]$. IL-21 can also promote IL-10 production, resulting in the inhibition of CD8 T cell activation and proliferation [49]. Thus, it is plausible that high levels of IL-21 may slow the differentiation of fullfledged effector CD8 T cells and favor memory CD8 T cell formation. The potential importance of IL-21 is further highlighted by studies using transgenic mice which overexpress IL21 , as this results in the accumulation of large numbers of memory phenotype CD8 $\mathrm{T}$ cells [50].

IL-21 also functions to regulate CD8 T cells at later stages and has been shown to inhibit $\mathrm{T}$ cell senescence, which in humans is characterized by the loss of CD28 expression, and contributes to age-associated defects in immunity. IL-21 blocks the downregulation of $\mathrm{CD} 28$ and also enhances the proliferation of CD28+ CD8 T cells [47,51,52]. Chronic viral infections can cause $\mathrm{T}$ cell exhaustion and, as discussed in Section 5, IL-21 directly acts on CD8 T cells to sustain their responsiveness under these conditions. IL-21 regulates multiple immune system components and therefore its effects on CD8 T cell responses may also be indirect. As previously outlined, IL-21 suppresses Foxp3+ regulatory CD4 T cells, and reductions in the numbers of suppressive Tregs can indirectly augment CD8 $\mathrm{T}$ cell responses [39]. Thus, the combined direct and indirect effects of IL-21 can shape the phenotype, functional quality, and sustainability of the CD8 T cell population.

\section{IL-21 and immunity to infections}

The severity of infections, as well as whether a pathogen will be cleared or will persist, is intimately coupled to both the innate and adaptive immune response. IL-21 can regulate these responses in multiple ways, as overviewed in Sections 3 and 4. Although the roles of IL-21 during immunity to infections are not currently extensively documented, a number of reports do implicate intriguing roles for this cytokine in dictating immunity to infections. The impact of IL-21 on antibody responses to pathogens is not well defined, but given the roles of IL-21 in promoting B cell responses and plasma cell formation, it is likely that humoral immunity to infections will be influenced by the availability and kinetics of induction of IL-21. The necessity for IL-21 in ensuring durable T cell immunity to acute infections appears to be subtle. Infection of IL-21R-/- mice with influenza virus or recombinant vaccinia virus encoding the lymphocytic choriomeningitis virus (LCMV) glycoprotein (VV-G2) has no effect on viral clearance or the frequency and function of the anti-viral $\mathrm{T}$ cells [11]. By contrast, infection of IL-21R-/- mice with a different vaccinia virus recombinant, expressing human immunodeficiency virus (HIV) gp160, has been reported to result in decreased cytolytic activity and weaker induction of the virus-specific $T$ cell response [46]. During acute LCMV infections reductions in the fraction of IL-2-producing virusspecific CD8 T cells have been observed in IL-21-/- mice and, under competitive conditions, recall responses are impaired if the responding cells do not express the IL-21R [48]. Unlike infections that are cleared, the emerging data set suggests that IL-21 is most critical during chronic infections, including infection of mice with certain strains of LCMV and also HIV infections of humans (Fig. 3).

\section{1. $L C M V \& I L-21$}

Studies of the immune response to LCMV have been instrumental in defining our understanding of immunity to infections. This natural mouse pathogen can cause rapidly resolved acute infections as well as more protracted and chronic infections, depending upon the strain of virus used and the age and immunological status of the host. Consequently, this system allows comparative analyses of the virological and immunological factors that are associated with these distinct patterns of infection. T cell exhaustion, which is characterized by the step-wise and progressive ablation of $\mathrm{T}$ cell functions and can culminate with the physical deletion of the responding cells, was discovered, and is most well defined, following LCMV infection [53]. In the absence of CD4 T cell help the ability to control the infection is further compromised, and severe CD8 T cell exhaustion can develop. Notably, under conditions of CD4 $\mathrm{T}$ cell deficiency the main producers of IL- 


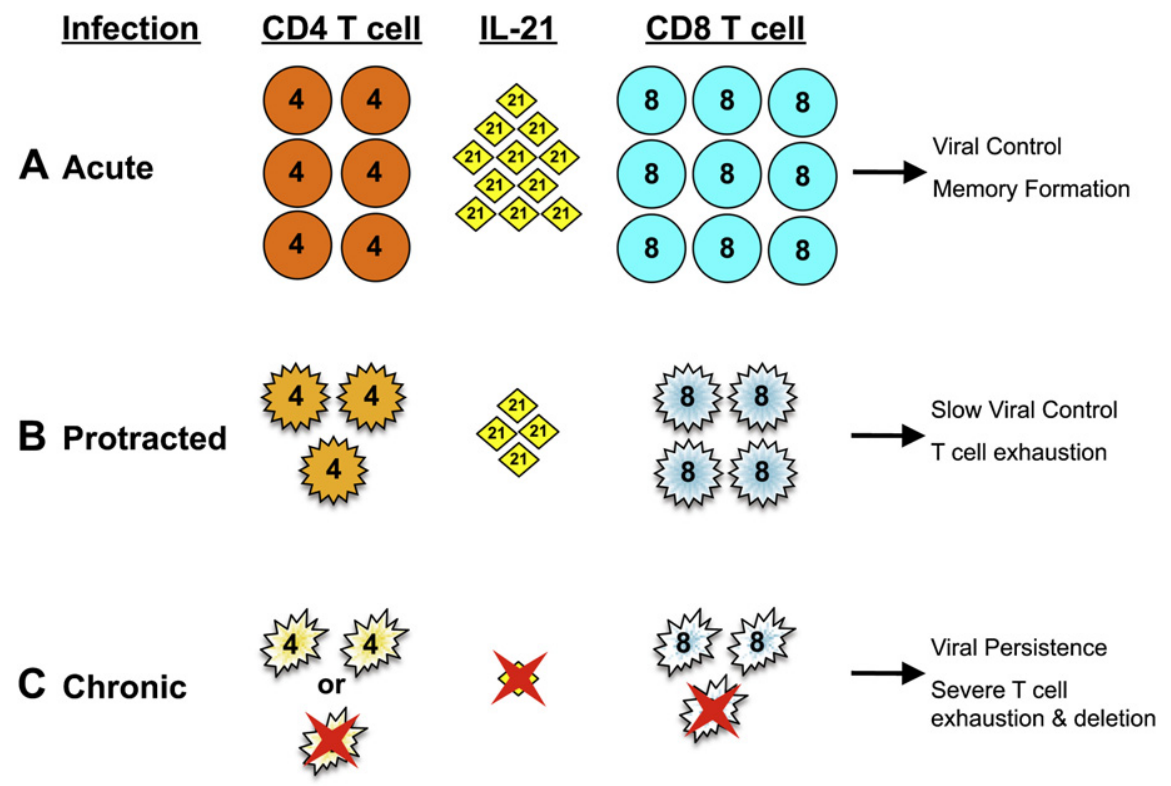

Fig. 3. CD4 T cell responses are a major determinant of IL-21 levels, which influence the quality of the CD8 T cell response and the control of viral infections. A. Acute viral infections commonly elicit IFN- $\gamma$ and IL-21 producing CD4 T cell responses. This is associated with robust effector CD8 T cell responses, memory formation, and control of the infection. B and C. Disruptions to the numbers and/or functional quality of CD4 T cell responses can result in diminished levels of IL21 producing cells, especially during the early stage of infection. In these circumstances, higher levels of virus replication, ablated CD4 T cell responses, as well as limited availability of IL-21 all likely contribute to impaired CD8 T cell responses and ineffective viral clearance.

21 are also absent, and several studies have now implicated IL21 as a vital helper factor which functions to sustain CD8 T cell responses during chronic LCMV infections $[11,54,55]$.

Acute LCMV infections elicit an initial increase in the levels of IL-21, principally due to activation of virus-specific CD4 T cells [11,48,54-56]. By contrast, CD4 T cell responses are markedly attenuated following inoculation with strains of LCMV which cause more protracted and chronic infections, and under these conditions the numbers of IL-21-producing CD4 $\mathrm{T}$ cells are lower, restricting the availability of IL-21 during the early stages of chronic LCMV infections (Fig. 3) [55]. Despite the lower numbers of virus-specific CD4 T cells capable of producing IL-21, chronic infections may result in upregulation of IL-21 levels in the CD4 T cells which are present, especially at later time points, possibly signifying a compensatory skewing of the response [54].

IL-21 has been implicated in driving the differentiation of CD4 T cells to Tfh lineage cells, which play a prominent role in promoting antibody responses, and has also been shown to help the formation of GC B cell reactions [29,30,41,42]. During acute LCMV infections Tfh (CXCR5+, ICOS+, PD$1+)$ and GC B cells (PNA+, CD19+, CD95+) are generated in the absence of IL-21, and LCMV-specific antibody titers are initially similar in wild-type, IL-21-/- and IL-21R-/- mice $[54,55]$. Nevertheless, at later time points antibody titers are somewhat reduced, which is consistent with additional findings documenting that the formation of GC B cells and Tfh CD4 T cells can occur independently of IL-21, but that IL-21 is more critically required for prolonging these responses $[29,30,41,42]$.

The importance of IL-21 in sustaining CD8 T cell responses during chronic LCMV infections has been further dissected using IL-21-/- and IL-21R-/- mice. Infection of these knockout mice with strains of LCMV that are predisposed to cause chronic infections results in severe CD8 T cell exhaustion, a phenotype which mirrors that observed following infection of CD4 $\mathrm{T}$ cell deficient hosts [11,54,55]. Notably, the induction of the primary LCMV-specific CD8 T cell response appears to proceed independently of IL-21, as fairly similar initial responses are elicited in the presence or absence of IL-21. The strict requirement for IL-21 manifests as the responses subsequently deteriorate in the IL-21-/- and IL-21R-/ - infected mice as severe CD8 T cell exhaustion sets in. This becomes apparent as effector activities, including the ability to produce IFN- $\gamma$, TNF- $\alpha$, and IL-2, are lost by the virus-specific CD8 $\mathrm{T}$ cells and the absolute numbers of these cells also decline $[11,54,55]$. In addition to the functional unresponsiveness that develops without IL-21 derived signals, the virus-specific $\mathrm{T}$ cells also adopt other phenotypic features of the exhausted state including the upregulation of CD43 (1B11 isoform) and maintained expression of the inhibitory receptor PD-1 [55]. Most significantly, in the absence of IL21 , infections with LCMV-clone 13 or high doses of the Docile strain are never brought under control $[11,54,55]$. Thus, the availability of IL-21 critically influences the functional quality and sustainability of CD8 T cell responses, especially under conditions of persistent antigenic activation which arise during chronic viral infections. This contrasts with responses to rapidly controlled infections, including acute LCMV infections, during which the requirements for IL-21 are less stringent.

As discussed in previous sections, IL-21 affects numerous aspects of the immune response. Therefore, to determine whether IL-21 was acting directly on virus-specific CD8 T 
cells, or indirectly through a third party, to sustain CD8 T cell responses during chronic LCMV infections, bone marrow chimeras were generated using a mixture of cells, which were (IL-21R+/+) and were not (IL-21R-/-) capable of receiving IL-21 dependent signals. Using this approach it was confirmed that primary LCMV-specific $\mathrm{T}$ cell responses were elicited independently of IL-21; however, it was further shown that subsequently the virus-specific IL-21R-/- CD8 T cells were at a selective disadvantage as these cells rapidly succumbed to exhaustion and were preferentially deleted $[11,54,55]$. The requirement for IL-21R expression by the responding virusspecific CD8 T cells demonstrates that the direct actions of IL21 on these cells are essential for sustaining responses during chronic infections.

The observations that the numbers of IL-21 producing CD4 T cells are reduced during the initial stages of more persistent infections, and that CD8 T cell exhaustion can develop if IL-21 is unavailable, indicates that the level of IL-21 influences the capacity to mount effective responses and contribute to infection control. It was therefore predicted that the administration of IL21 would have a positive impact on the quality of the CD8 T cell response and accelerate viral clearance. Administration of recombinant IL-21 between days $0-8$ following LCMV-clone 13 infection of CD4-/- mice reduced exhaustion and enhanced viral control [55]. Although IL-21 therapy helped prevent the development of exhaustion and resulted in lower viral loads, many of the mice undergoing treatment became moribund, probably due to immunopathology caused by the enhanced CD8 $\mathrm{T}$ cell response. This illustrates the role of $\mathrm{T}$ cell exhaustion as a regulatory mechanism for attenuating possibly pathogenic immune responses to persistent infections. Important next steps will be to use our improved understanding of the roles of IL-21 to devise approaches, such as combination therapies, that promote anti-viral responses and containment of the infection, while avoiding detrimental effects such as enhanced immunopathology.

\subsection{HIV and IL-21}

HIV replicates in CD4 T cells and upon infection causes a profound initial depletion of these cells. If left untreated HIV infection typically results in a continued erosion of CD4 T cell numbers and progression to AIDS. Infection-associated disruptions to the CD4 $\mathrm{T}$ cell population are the major reason for the broad immunological defects which develop in HIV positive individuals. Since CD4 T cells are the main source of IL-21, there is interest in determining whether and how HIV infection alters IL-21 production, and in defining the consequences of these changes on immunological functions and disease progression [57,58]. Lower IL-21 levels have been detected in the serum of HIV-infected individuals by comparison with non-infected control subjects [57]. These studies also show that the levels of IL-21 correlate with CD4 T cell counts, which is consistent with CD4 $\mathrm{T}$ cells being the most significant producers of IL-21. Further stratification of the subjects based on viral loads revealed an inverse correlation between IL-21 levels and the ability to contain the infection, with the highest IL-21 levels detected in patients with low viral loads [57,58]. The application of anti-viral therapy decreased viral replication and the resulting increase in $\mathrm{CD} 4 \mathrm{~T}$ cell counts was associated with a concordant increase in IL-21 levels. Elite controllers, which are able to contain HIV infection without treatment, also have relatively high levels of IL-21 in the circulation. Further analyses of IL21 production by CD4 $\mathrm{T}$ cells from HIV-infected patients, as well as in vitro studies, suggest that the ability of CD4 T cells to synthesize IL-21 is specifically reduced upon HIV infection. The downregulation is at least in part attributable to modulation of c-Maf, a transcription factor required for activation of IL-21 expression [57]. Inhibiting c-Maf expression using siRNA reduces the production of IL-21 and IL-4, but not IL-2, by the CD4 $\mathrm{T}$ cells.

During HIV infection the presence and functionality of virus-specific CD8 $\mathrm{T}$ cells help to keep the infection in check. Thus, the preservation of these responses can result in lower viral set points and slower disease progression. As discussed above, in mice chronically infected with LCMV, IL-21 has been shown to profoundly impact the virus-specific $\mathrm{T}$ cell response. During HIV-1 infection IL-21 levels also potentially influence the phenotypic and functional properties of anti-viral CD8 T cells. Notably, functional HIV-specific CD8 T cell responses, as detected by the ability to produce IL-2 and IFN$\gamma$, tend to be greater in individuals with high serum levels of IL-21 [57]. HIV-specific CD8 T cells from chronically infected individuals have also been shown to express higher levels of the IL-21R than their cytomegalovirus (CMV)-specific counterparts, and culturing in the presence of IL-21 promotes the survival and expansion of these cells [57,58].

IL-21 also impacts other functional attributes of CD8 T cells which affects their ability to contain infections. Perforin is a key molecule deployed by CD8 T cells to kill virus-infected target cells, and perforin-dependent killing functions are important for maintaining viral-loads at a steady state level. Ex vivo treatment of purified T cells from HIV-infected donors with IL-21 causes a selective increase in perforin expression but does not drive the proliferation or the degranulation of the $\mathrm{CD} 8 \mathrm{~T}$ cell population, which are instead promoted by IL-15 [59]. Therefore, the elevation of perforin levels can be uncoupled from cell proliferation. Thus, IL-21 may function to increase the per cell killing efficacy of individual CD8 T cells, which may improve overall viral containment.

Granulysin is expressed by CD8 T cells and NK cells in humans and has anti-microbial activity against pathogens such as Mycobacterium tuberculosis. Consequently, this effector mechanism contributes to resistance to certain opportunistic infections which develop in immune compromised individuals. IL-21 and IL-15 have been shown to increase granulysin levels in CD45RO+ CD8 T cells from HIV-negative donors and is associated with STAT3 and STAT5 activation [60]. Infection of peripheral blood mononuclear cells (PBMCs) with HIV significantly reduces IL-21-induced granulysin and perforin production by CD8 T cells, but the inhibition is indirect, possibly due to a suppressive soluble factor secreted by the infected CD4 $\mathrm{T}$ cells in the bulk cultures. Collectively, these 
studies reveal pivotal, but potentially subtle, roles for IL-21 in shaping the functional properties of CD8 $\mathrm{T}$ cell responses during HIV-1 infection and also show that these qualities can be further influenced by the underlying infection.

Innate immunity provides critical initial defense against pathogens, including HIV, and the capacity to achieve very early viral control may positively impact the subsequent course of the infection. NK cells are prominent components of the innate arm of the immune response. NK cells can be categorized by the levels of expression of CD16 and CD56, as well as by their capacity to kill target cells and produce cytokines. Typically NK cells in the circulation are either CD56dim, CD16+ or CD56bright, CD16-, but during HIV infection shifts occur in the NK cell population as a subset of anergic CD56-, CD16+ cells emerge [61]. All of these subsets express the IL-21R and can be influenced by in vitro exposure to IL-21. Interestingly, the CD56dim subset of NK cells, which are preferentially dependent upon IL-21, have been reported to be reduced during HIV infection [62]. IL-21 enhances the responses of NK cells from HIV-infected subjects by stimulating perforin production [62]. This IL-21 induced increase in cytolytic potential is STAT-3 dependent as the STAT-3 inhibitor cucuribit blocks the augmentation of the killing activity of IL-21 treated NK cells [63]. NK cells stimulated with IL-21 also exhibit enhanced antibody-dependent cell-mediated cytoxicity against antibody-coated target cells and are also more effective at suppressing HIV replication in vitro [63]. Although IL-21 alone does not promote marked proliferation of NK cell subsets, it does function as a prosurvival factor by inducing the expression of the antiapoptotic proteins $\mathrm{Bcl}-2$ and $\mathrm{Bcl}-\mathrm{X}_{\mathrm{L}}$.

Chronic HIV infection, as well as disease susceptibility in non-human primates, is associated with a preferential loss of Th17 cells at mucosal sites and generalized immune activation $[64,65]$. Imbalances in the ratio of Th17:Tregs have also been reported, with declines in the Th17 population but a relative increase in the frequencies of Tregs correlating with more pronounced immune activation [64]. The contributions of IL21 in controlling equilibrium of Treg and Th17 responses during HIV and SIV infections have not yet been documented. Nevertheless, given the roles of IL-21 in both promoting Th17 development and in suppressing Treg levels, it is possible that alterations in the availability of IL-21 in the intestinal mucosa, in addition to the overall systemic levels, have profound effects on HIV disease progression and pathogenesis. Further investigations will be necessary to define these potential aspects of IL-21.

\subsection{Helminth infections}

As outlined in Section 4.2, IL-21 plays multifaceted roles in influencing the differentiation of naïve CD4 $\mathrm{T}$ cells into functionally distinct subsets. Studies of mice infected with the helminths Nippostrongylus brasiliensis, Heligmosomoides polygyrus, and Schistosoma mansoni have documented the importance of IL-21 in establishing Th2 mediated immunity $[43,44,66,67]$.
Infection of IL-21R -/ - mice with $N$. brasiliensis resulted in the reduction of eosinophils, lymphocytes, and macrophages in addition to lower levels of IgA and IgG1 in lung lavages [67]. Diminished Th2 associated responses were similarly noted in the absence of IL-21 signaling following intestinal infection with $H$. polygyrus [67]. IL-21 is not necessarily required for Th2 differentiation, but IL-21R-deficient Th2 CD4 $\mathrm{T}$ cells exhibited impaired expansion or survival, which results in a lower overall inflammatory response at local sites of infection [66,67]. The direct requirement for IL-21 to support developing Th2 CD4 T cells is likely responsible for the reduced responses.

IL-21 has been shown to play a role in sustaining Tfh responses, and in the context of parasitic infections, interrelationships between $\mathrm{Th} 2$ responses and Tfh cells have been noted. Tfh cells have been shown to develop from Th2 cells following $S$. mansoni and $H$. polygyrus infection, and Tfh cells have also been shown to be a major source of IL-4 $[43,44]$. The precise roles of IL-21 in determining the relative abundance and balance of Th2 and Tfh responses as well as the nuances of their developmental connections are not yet well defined.

\section{Conclusions}

As discussed throughout this article, IL-21 has multiple roles in regulating both innate and adaptive immune responses and has the potential to impact many aspects of the immunemediated control of infections. The studies of LCMV infection of mice, as well as HIV infection, especially implicate the importance IL-21 in promoting and sustaining immunity to chronic infections. It will therefore be interesting to discover whether IL-21 plays significant roles in determining the outcomes of other chronic infections, such as hepatitis $\mathrm{C}$ virus. Moreover, whether IL-21 is more selectively required to support responses to high-grade chronic infections or also contributes to the control of latent infections, which are prone to period reactivation, is not yet defined. Since IL-21 has also been shown to impede $\mathrm{T}$ cell senescence, it will be of interest to investigate whether changes in the availability of IL-21 contributes to age-associated changes in susceptibility to infections.

The pleiotropic effects of IL-21 complicate the analyses of the precise role of this cytokine in the immune responses to infections. Thus, it remains necessary to further dissect how this cytokine interconnects with other immune system components, including other $\gamma c$ cytokines, over the course of an infection, and define how these interactions shape effectiveness of the immune response. Understanding these processes will provide fundamental insights into how IL-21 impacts the induction, quality, and maintenance of responses to infections. This information, in conjunction with knowledge gained from the analysis of the influence of IL-21 on autoimmunity and immunity to tumors, may then advance the development of IL-21 based adjuvants or therapies that could be used as stand-alone approaches or in combination with other treatment strategies. 


\section{Acknowledgements}

We wish to thank Laurie Harrington and all of the members of the Zajac laboratory for their advice and critical reading of this manuscript. This work was supported in part by grants R01 AI049360, R01 AI067933, and U01 AI082966 (to A.J.Z) and T32 AI007051 (to J.S.Y) from the National Institutes of Health. We regret that due to space limitations, we were unable to cite all our colleagues who have contributed to our current knowledge of IL-21.

\section{References}

[1] D.S. Mehta, A.L. Wurster, M.J. Grusby, Biology of IL-21 and the IL-21 receptor, Immunol. Rev. 202 (2004) 84-95.

[2] R. Spolski, W.J. Leonard, Interleukin-21: basic biology and implications for cancer and autoimmunity, Annu. Rev. Immunol. 26 (2008) 57-79.

[3] K. Ozaki, K. Kikly, D. Michalovich, P.R. Young, W.J. Leonard, Cloning of a type I cytokine receptor most related to the IL-2 receptor beta chain, Proc. Natl. Acad. Sci. U.S.A. 97 (2000) 11439-11444.

[4] J. Parrish-Novak, S.R. Dillon, A. Nelson, A. Hammond, C. Sprecher, J.A. Gross, J. Johnston, K. Madden, W. Xu, J. West, S. Schrader, S. Burkhead, M. Heipel, C. Brandt, J.L. Kuijper, J. Kramer, D. Conklin, S.R. Presnell, J. Berry, F. Shiota, S. Bort, K. Hambly, S. Mudri, C. Clegg, M. Moore, F.J. Grant, C. Lofton-Day, T. Gilbert, F. Rayond, A. Ching, L. Yao, D. Smith, P. Webster, T. Whitmore, M. Maurer, K. Kaushansky, R.D. Holly, D. Foster, Interleukin 21 and its receptor are involved in NK cell expansion and regulation of lymphocyte function, Nature 408 (2000) 57-63.

[5] H. Asao, C. Okuyama, S. Kumaki, N. Ishii, S. Tsuchiya, D. Foster, K. Sugamura, Cutting edge: the common gamma-chain is an indispensable subunit of the IL-21 receptor complex, J. Immunol. 167 (2001) 1-5.

[6] K. Brandt, S. Bulfone-Paus, A. Jenckel, D.C. Foster, R. Paus, R. Ruckert, Interleukin-21 inhibits dendritic cell-mediated $\mathrm{T}$ cell activation and induction of contact hypersensitivity in vivo, J. Invest. Dermatol. 121 (2003) 1379-1382.

[7] G. Monteleone, R. Caruso, D. Fina, I. Peluso, V. Gioia, C. Stolfi, M.C. Fantini, F. Caprioli, R. Tersigni, L. Alessandroni, T.T. MacDonald, F. Pallone, Control of matrix metalloproteinase production in human intestinal fibroblasts by interleukin 21, Gut 55 (2006) 1774-1780.

[8] R. Caruso, D. Fina, I. Peluso, M.C. Fantini, C. Tosti, G. Del Vecchio Blanco, O.A. Paoluzi, F. Caprioli, F. Andrei, C. Stolfi, M. Romano, V. Ricci, T.T. MacDonald, F. Pallone, G. Monteleone, IL-21 is highly produced in Helicobacter pylori-infected gastric mucosa and promotes gelatinases synthesis, J. Immunol. 178 (2007) 5957-5965.

[9] R. Ruckert, S. Bulfone-Paus, K. Brandt, Interleukin-21 stimulates antigen uptake, protease activity, survival and induction of $\mathrm{CD} 4+\mathrm{T}$ cell proliferation by murine macrophages, Clin. Exp. Immunol. 151 (2008) $487-495$.

[10] J.M. Coquet, K. Kyparissoudis, D.G. Pellicci, G. Besra, S.P. Berzins, M.J. Smyth, D.I. Godfrey, IL-21 is produced by NKT cells and modulates NKT cell activation and cytokine production, J. Immunol. 178 (2007) 2827-2834.

[11] A. Frohlich, J. Kisielow, I. Schmitz, S. Freigang, A.T. Shamshiev, J. Weber, B.J. Marsland, A. Oxenius, M. Kopf, IL-21R on T cells is critical for sustained functionality and control of chronic viral infection, Science 324 (2009) 1576-1580.

[12] C. Ortega, A.S. Fernandez, J.M. Carrillo, P. Romero, I.J. Molina, J.C. Moreno, M. Santamaria, IL-17-producing CD8+ T lymphocytes from psoriasis skin plaques are cytotoxic effector cells that secrete Th17related cytokines, J. Leukoc. Biol. 86 (2009) 435-443.

[13] K. Ozaki, R. Spolski, R. Ettinger, H.P. Kim, G. Wang, C.F. Qi, P. Hwu, D. J. Shaffer, S. Akilesh, D.C. Roopenian, H.C. Morse 3rd, P.E. Lipsky, W. J. Leonard, Regulation of B cell differentiation and plasma cell generation by IL-21, a novel inducer of Blimp-1 and Bcl-6, J. Immunol. 173 (2004) 5361-5371.
[14] C.S. Hinrichs, R. Spolski, C.M. Paulos, L. Gattinoni, K.W. Kerstann, D. C. Palmer, C.A. Klebanoff, S.A. Rosenberg, W.J. Leonard, N.P. Restifo, IL-2 and IL-21 confer opposing differentiation programs to CD8+ T cells for adoptive immunotherapy, Blood 111 (2008) 5326-5333.

[15] A.T. Bauquet, H. Jin, A.M. Paterson, M. Mitsdoerffer, I.C. Ho, A.H. Sharpe, V.K. Kuchroo, The costimulatory molecule ICOS regulates the expression of c-Maf and IL-21 in the development of follicular T helper cells and TH-17 cells, Nat. Immunol. 10 (2009) 167-175.

[16] H. Kwon, D. Thierry-Mieg, J. Thierry-Mieg, H.P. Kim, J. Oh, C. Tunyaplin, S. Carotta, C.E. Donovan, M.L. Goldman, P. Tailor, K. Ozato, D.E. Levy, S.L. Nutt, K. Calame, W.J. Leonard, Analysis of interleukin-21-induced Prdm1 gene regulation reveals functional cooperation of STAT3 and IRF4 transcription factors, Immunity 31 (2009) 941-952.

[17] M. Strengell, A. Lehtonen, S. Matikainen, I. Julkunen, IL-21 enhances SOCS gene expression and inhibits LPS-induced cytokine production in human monocyte-derived dendritic cells, J. Leukoc. Biol. 79 (2006) 1279-1285.

[18] M.T. Kasaian, M.J. Whitters, L.L. Carter, L.D. Lowe, J.M. Jussif, B. Deng, K.A. Johnson, J.S. Witek, M. Senices, R.F. Konz, A.L. Wurster, D.D. Donaldson, M. Collins, D.A. Young, M.J. Grusby, IL-21 limits NK cell responses and promotes antigen-specific $\mathrm{T}$ cell activation: a mediator of the transition from innate to adaptive immunity, Immunity 16 (2002) 559-569.

[19] J. Brady, Y. Hayakawa, M.J. Smyth, S.L. Nutt, IL-21 induces the functional maturation of murine NK cells, J. Immunol. 172 (2004) 2048-2058.

[20] S. Sivori, C. Cantoni, S. Parolini, E. Marcenaro, R. Conte, L. Moretta, A. Moretta, IL-21 induces both rapid maturation of human CD34+ cell precursors towards NK cells and acquisition of surface killer Ig-like receptors, Eur. J. Immunol. 33 (2003) 3439-3447.

[21] M. Strengell, S. Matikainen, J. Siren, A. Lehtonen, D. Foster, I. Julkunen, T. Sareneva, IL-21 in synergy with IL-15 or IL-18 enhances IFN-gamma production in human NK and T cells, J. Immunol. 170 (2003) 5464-5469.

[22] K. Wendt, E. Wilk, S. Buyny, R.E. Schmidt, R. Jacobs, Interleukin-21 differentially affects human natural killer cell subsets, Immunology 122 (2007) 486-495.

[23] M.J. Smyth, M.E. Wallace, S.L. Nutt, H. Yagita, D.I. Godfrey, Y. Hayakawa, Sequential activation of NKT cells and NK cells provides effective innate immunotherapy of cancer, J. Exp. Med. 201 (2005) 1973-1985.

[24] D.S. Mehta, A.L. Wurster, M.J. Whitters, D.A. Young, M. Collins, M.J. Grusby, IL-21 induces the apoptosis of resting and activated primary B cells, J. Immunol. 170 (2003) 4111-4118.

[25] H. Jin, R. Carrio, A. Yu, T.R. Malek, Distinct activation signals determine whether IL-21 induces B cell costimulation, growth arrest, or Bimdependent apoptosis, J. Immunol. 173 (2004) 657-665.

[26] K. Ozaki, R. Spolski, C.G. Feng, C.F. Qi, J. Cheng, A. Sher, H.C. Morse 3rd, C. Liu, P.L. Schwartzberg, W.J. Leonard, A critical role for IL-21 in regulating immunoglobulin production, Science 298 (2002) $1630-1634$.

[27] R.J. Johnston, A.C. Poholek, D. DiToro, I. Yusuf, D. Eto, B. Barnett, A.L. Dent, J. Craft, S. Crotty, Bcl6 and Blimp-1 are reciprocal and antagonistic regulators of $\mathrm{T}$ follicular helper cell differentiation, Science 325 (2009) 1006-1010.

[28] R.I. Nurieva, Y. Chung, G.J. Martinez, X.O. Yang, S. Tanaka, T.D Matskevitch, Y.H. Wang, C. Dong, Bcl6 mediates the development of T follicular helper cells, Science 325 (2009) 1001-1005.

[29] M.A. Linterman, L. Beaton, D. Yu, R.R. Ramiscal, M. Srivastava, J.J. Hogan, N.K. Verma, M.J. Smyth, R.J. Rigby, C.G. Vinuesa, IL-21 acts directly on B cells to regulate Bcl-6 expression and germinal center responses, J. Exp. Med. 207 (2010) 353-363.

[30] D. Zotos, J.M. Coquet, Y. Zhang, A. Light, K. D'Costa, A. Kallies, L.M. Corcoran, D.I. Godfrey, K.M. Toellner, M.J. Smyth, S.L. Nutt, D.M. Tarlinton, IL-21 regulates germinal center B cell differentiation and proliferation through a B cell-intrinsic mechanism, J. Exp. Med. 207 (2010) 365-378.

[31] R. Ettinger, G.P. Sims, A.M. Fairhurst, R. Robbins, Y.S. da Silva, R. Spolski, W.J. Leonard, P.E. Lipsky, IL-21 induces differentiation of 
human naive and memory B cells into antibody-secreting plasma cells, J. Immunol. 175 (2005) 7867-7879.

[32] V.L. Bryant, C.S. Ma, D.T. Avery, Y. Li, K.L. Good, L.M. Corcoran, R. de Waal Malefyt, S.G. Tangye, Cytokine-mediated regulation of human B cell differentiation into Ig-secreting cells: predominant role of IL-21 produced by CXCR5+ T follicular helper cells, J. Immunol. 179 (2007) 8180-8190.

[33] S.A. Diehl, H. Schmidlin, M. Nagasawa, S.D. van Haren, M.J. Kwakkenbos, E. Yasuda, T. Beaumont, F.A. Scheeren, H. Spits, STAT3mediated up-regulation of BLIMP1 Is coordinated with BCL6 downregulation to control human plasma cell differentiation, J. Immunol. 180 (2008) 4805-4815.

[34] L. Zhou, I.I. Ivanov, R. Spolski, R. Min, K. Shenderov, T. Egawa, D.E. Levy, W.J. Leonard, D.R. Littman, IL-6 programs T(H)-17 cell differentiation by promoting sequential engagement of the IL-21 and IL-23 pathways, Nat. Immunol. 8 (2007) 967-974.

[35] T. Korn, E. Bettelli, W. Gao, A. Awasthi, A. Jager, T.B. Strom, M. Oukka, V.K. Kuchroo, IL-21 initiates an alternative pathway to induce proinflammatory $\mathrm{T}(\mathrm{H}) 17$ cells, Nature 448 (2007) 484-487.

[36] R. Nurieva, X.O. Yang, G. Martinez, Y. Zhang, A.D. Panopoulos, L. Ma, K. Schluns, Q. Tian, S.S. Watowich, A.M. Jetten, C. Dong, Essential autocrine regulation by IL-21 in the generation of inflammatory T cells, Nature 448 (2007) 480-483.

[37] L. Yang, D.E. Anderson, C. Baecher-Allan, W.D. Hastings, E. Bettelli, M. Oukka, V.K. Kuchroo, D.A. Hafler, IL-21 and TGF-beta are required for differentiation of human T(H)17 cells, Nature 454 (2008) 350-352.

[38] A. Suto, D. Kashiwakuma, S. Kagami, K. Hirose, N. Watanabe, K. Yokote, Y. Saito, T. Nakayama, M.J. Grusby, I. Iwamoto, H. Nakajima, Development and characterization of IL-21-producing CD4+ T cells, J. Exp. Med. 205 (2008) 1369-1379.

[39] Y. Li, C. Yee, IL-21 mediated Foxp3 suppression leads to enhanced generation of antigen-specific CD8+ cytotoxic T lymphocytes, Blood 111 (2008) 229-235.

[40] C. King, S.G. Tangye, C.R. Mackay, T follicular helper (TFH) cells in normal and dysregulated immune responses, Annu. Rev. Immunol. 26 (2008) 741-766.

[41] R.I. Nurieva, Y. Chung, D. Hwang, X.O. Yang, H.S. Kang, L. Ma, Y.H. Wang, S.S. Watowich, A.M. Jetten, Q. Tian, C. Dong, Generation of T follicular helper cells is mediated by interleukin-21 but independent of T helper 1, 2, or 17 cell lineages, Immunity 29 (2008) 138-149.

[42] A. Vogelzang, H.M. McGuire, D. Yu, J. Sprent, C.R. Mackay, C. King, A fundamental role for interleukin-21 in the generation of $\mathrm{T}$ follicular helper cells, Immunity 29 (2008) 127-137.

[43] I.L. King, M. Mohrs, IL-4-producing CD4+ T cells in reactive lymph nodes during helminth infection are T follicular helper cells, J. Exp. Med. 206 (2009) 1001-1007.

[44] A.G. Zaretsky, J.J. Taylor, I.L. King, F.A. Marshall, M. Mohrs, E.J. Pearce, $\mathrm{T}$ follicular helper cells differentiate from Th2 cells in response to helminth antigens, J. Exp. Med. 206 (2009) 991-999.

[45] K.A. Casey, M.F. Mescher, IL-21 promotes differentiation of naive CD8 T cells to a unique effector phenotype, J. Immunol. 178 (2007) 7640-7648.

[46] R. Zeng, R. Spolski, S.E. Finkelstein, S. Oh, P.E. Kovanen, C.S. Hinrichs, C.A. Pise-Masison, M.F. Radonovich, J.N. Brady, N.P. Restifo, J.A. Berzofsky, W.J. Leonard, Synergy of IL-21 and IL-15 in regulating CD8+ T cell expansion and function, J. Exp. Med. 201 (2005) 139-148.

[47] Y. Li, M. Bleakley, C. Yee, IL-21 influences the frequency, phenotype, and affinity of the antigen-specific CD8 T cell response, J. Immunol. 175 (2005) 2261-2269.

[48] J.S. Yi, J.T. Ingram, A.J. Zajac, IL-21-deficiency influences CD8 T Cell quality and recall responses following an acute viral infection, J. Immunol. 185 (2010), doi:10.4049/jimmunol.1001032.

[49] R. Spolski, H.P. Kim, W. Zhu, D.E. Levy, W.J. Leonard, IL-21 mediates suppressive effects via its induction of IL-10, J. Immunol. 182 (2009) 2859-2867.
[50] E.L. Allard, M.P. Hardy, J. Leignadier, M. Marquis, J. Rooney, D. Lehoux, N. Labrecque, Overexpression of IL-21 promotes massive CD8+ memory T cell accumulation, Eur. J. Immunol. 37 (2007) 3069-3077.

[51] N.L. Alves, F.A. Arosa, R.A. van Lier, IL-21 sustains CD28 expression on IL-15-activated human naive CD8+ T cells, J. Immunol. 175 (2005) $755-762$.

[52] H. Nguyen, N.P. Weng, IL-21 preferentially enhances IL-15-mediated homeostatic proliferation of human CD28+ CD8 memory $\mathrm{T}$ cells throughout the adult age span, J. Leukoc. Biol. 87 (2010) 43-49.

[53] J.S. Yi, M.A. Cox, A.J. Zajac, T-cell exhaustion: characteristics, causes and conversion, Immunology 129 (2010) 474-481.

[54] H. Elsaesser, K. Sauer, D.G. Brooks, IL-21 is required to control chronic viral infection, Science 324 (2009) 1569-1572.

[55] J.S. Yi, M. Du, A.J. Zajac, A vital role for interleukin-21 in the control of a chronic viral infection, Science 324 (2009) 1572-1576.

[56] C. Holm, C.G. Nyvold, S.R. Paludan, A.R. Thomsen, M. Hokland, Interleukin-21 mRNA expression during virus infections, Cytokine 33 (2006) 41-45.

[57] A. Iannello, M.R. Boulassel, S. Samarani, O. Debbeche, C. Tremblay, E. Toma, J.P. Routy, A. Ahmad, Dynamics and consequences of IL-21 production in HIV-infected individuals: a longitudinal and crosssectional study, J. Immunol. 184 (2010) 114-126.

[58] F.Y. Yue, C. Lo, A. Sakhdari, E.Y. Lee, C.M. Kovacs, E. Benko, J. Liu, H. Song, R.B. Jones, P. Sheth, D. Chege, R. Kaul, M.A. Ostrowski, HIVspecific IL-21 producing $\mathrm{CD} 4(+) \mathrm{T}$ cells are induced in acute and chronic progressive HIV infection and are associated with relative viral control, J. Immunol. 185 (2010) 498-506.

[59] L. White, S. Krishnan, N. Strbo, H. Liu, M.A. Kolber, M.G. Lichtenheld, R.N. Pahwa, S. Pahwa, Differential effects of IL-21 and IL-15 on perforin expression, lysosomal degranulation, and proliferation in CD8 T cells of patients with human immunodeficiency virus-1 (HIV), Blood 109 (2007) 3873-3880.

[60] A.E. Hogg, G.C. Bowick, N.K. Herzog, M.W. Cloyd, J.J. Endsley, Induction of granulysin in CD8+ T cells by IL-21 and IL-15 is suppressed by human immunodeficiency virus-1, J. Leukoc. Biol. 86 (2009) 1191-1203.

[61] G. Alter, M. Altfeld, NK cells in HIV-1 infection: evidence for their role in the control of HIV-1 infection, J. Intern. Med. 265 (2009) 29-42.

[62] N. Strbo, L. de Armas, H. Liu, M.A. Kolber, M. Lichtenheld, S. Pahwa, IL-21 augments natural killer effector functions in chronically HIVinfected individuals, AIDS 22 (2008) 1551-1560.

[63] A. Iannello, M.R. Boulassel, S. Samarani, C. Tremblay, E. Toma, J.P. Routy, A. Ahmad, IL-21 enhances NK cell functions and survival in healthy and HIV-infected patients with minimal stimulation of viral replication, J. Leukoc. Biol. (2010).

[64] D. Favre, S. Lederer, B. Kanwar, Z.M. Ma, S. Proll, Z. Kasakow, J. Mold, L. Swainson, J.D. Barbour, C.R. Baskin, R. Palermo, I. Pandrea, C.J. Miller, M.G. Katze, J.M. McCune, Critical loss of the balance between Th17 and T regulatory cell populations in pathogenic SIV infection, PLoS Pathog. 5 (2009) e1000295.

[65] J.M. Brenchley, M. Paiardini, K.S. Knox, A.I. Asher, B. Cervasi, T.E. Asher, P. Scheinberg, D.A. Price, C.A. Hage, L.M. Kholi, A. Khoruts, I. Frank, J. Else, T. Schacker, G. Silvestri, D.C. Douek, Differential Th17 CD4 T-cell depletion in pathogenic and nonpathogenic lentiviral infections, Blood 112 (2008) 2826-2835.

[66] J. Pesce, M. Kaviratne, T.R. Ramalingam, R.W. Thompson, J.F. Urban Jr. , A.W. Cheever, D.A. Young, M. Collins, M.J. Grusby, T.A. Wynn, The IL-21 receptor augments Th2 effector function and alternative macrophage activation, J. Clin. Invest. 116 (2006) 2044-2055.

[67] A. Frohlich, B.J. Marsland, I. Sonderegger, M. Kurrer, M.R. Hodge, N.L. Harris, M. Kopf, IL-21 receptor signaling is integral to the development of Th2 effector responses in vivo, Blood 109 (2007) 2023-2031. 